\title{
COSMIC-RAY SELF-CONFINEMENT IN THE HOT PHASE OF THE INTERSTELLAR MEDIUM
}

\author{
Catherine J. Cesarsky \\ Section d'Astrophysique \\ Centre d'Etudes Nucléaires de Saclay, France
}

\section{Russell M. Kulsrud \\ Plasma Physics Laboratory, Princeton, New Jersey}

It is well known that, when cosmic rays stream along the field lines of the galactic magnetic field at a velocity which exceeds the Alf ven velocity in the medium, they excite hydromagnetic waves of a wavelength comparable to their Larmor radius. These waves, in turn, scatter the cosmic rays, forcing them to reduce their bulk speed (Wentzel 1974, and references there-in). In a stationary situation, the bulk speed of the cosmic rays will depend on their scale height, and on the strength of the relevant damping mechanisms affecting the waves. Isotope observations imply that cosmic rays of energy $\leqslant 1 \mathrm{GeV}$ are confined in the galaxy for a time $\sim 2.10^{7}$ yrs (Garcia-Munoz et al. 1977); a simple interpretation of the composition data in the energy range $1-100 \mathrm{GeV}$ implies that the confinement time $T$ decreases as the rigidity $\varepsilon=c p / e Z$ increases: $T \alpha \varepsilon^{-\mathrm{a}}$, with $\mathrm{a}=0.3-0.5$. This energy dependence of the confinement time should hold at least up to $\varepsilon \simeq 3.10^{6} \mathrm{GV}$, to be consistent with the lack of structure in the cosmic ray spectrum in the range $10-3.10^{6} \mathrm{GV}$ (see Cesarsky 1980 and references there in).

Until a few years ago, it was believed that the interstellar medium was mostly filled by a neutral gas, of density $\sim 0.1 \mathrm{~cm}^{-3}$ and a temperature of several thousand degrees. Kulsrud and Cesarsky (1971) showed that, in such a medium, cosmic rays of energy $\geqslant 100 \mathrm{GeV}$ are not confined at all, because the waves are damped very rapidly by the effect of the collisions between the neutral and the charged particles in the medium. The case of streaming in HII regions was considered by Wentzel (1974) and Skilling (1975), and did not lead either to a satisfactory solution.

At present, we think that a substantial fraction of the interstellar medium is filled with a hot $\left(T_{6} \simeq 1\right.$, where $T_{6}$ is the temperature in $\left.10^{6}{ }^{\circ} \mathrm{K}\right)$ and diffuse "coronal gas" $\left(n-3 \simeq 1\right.$, where $n-3$ is the density in $\left.10^{-3} \mathrm{~cm}^{-3}\right)$. The strength of the magnetic field in such regions is unknown; it is probably lower than the "normal" interstellar value, $2.5 \mu \mathrm{G}$, by a factor which may be in the range 3-30. If $B_{\mu}$ is the magnetic field strength in $\mu$-gauss, the Alfven velocity is $v_{A}=66 B_{\mu} /(n-3)^{0.5} \mathrm{Km} \mathrm{s}^{-1}$, while the thermal velocity is $v_{i}=156\left(\mathrm{~T}_{6}\right)^{0.5} \mathrm{Km} \mathrm{s}^{-1}$. The main damping mechanisms affecting hydromagnetic waves in $\mathrm{HI}$ and HII regions are not operative in the coronal medium, where neutral particles are absent, and where, with $v_{i}>v_{A}$, Alfven waves cannot decay via two-wave interactions into sound waves. Still, waves propagating at an angle with the magnetic field are rapidly damped by 
collisionless processes while waves propagating along the field, but whose frequency is higher than the Larmor frequency of the thermal ions, are dissipated by Landau damping. However, all these "usual" damping mechanisms do not affect the Alfven waves propagating along the magnetic field, and resonating with cosmic rays ; thus the "coronal" phase of the interstellar medium appears to be a prime candidate as a region for cosmic ray self-confinement (Mc Cray and Snow 1979).

In fact, as pointed out by Lee and Völk (1973) and Kulsrud (1978), parallel propagating Alfven waves in hot plasma are also subjected to a non-linear damping mechanism, which is due to interactions of the thermal ions with beat waves produced by couples of Alfven waves. The non-linear damping rate of waves resonating with cosmic rays of Larmor frequency $\Omega$ is estimated by Kulsrud (1978) as :

$$
\Gamma_{n \ell} \sim(\sqrt{\pi} / 8)\left(v_{i} / c\right)(\delta B / B)^{2} \Omega
$$

where $(\delta B / B)^{2}$ represents the ratio of the energy density in resonant waves to that in the underlying magnetic field.

Setting the growth rate equal to the damping rate, we obtain the streaming velocity $\Delta \mathbf{v}$ of cosmic rays of rigidity $\varepsilon$ with respect to a frame moving at the Alfven speed

$$
\Delta v \simeq 10 \varepsilon^{0.85}\left(\mathrm{~T}_{6} \mathrm{n}_{-3}\right)^{0.25} \mathrm{~L}_{1}^{-0.5} \mathrm{Km} \mathrm{s}^{-1}
$$

where $L_{1}$ (Kpsec) is the scale height of cosmic rays along the magnetic flux tubes, and for a cosmic ray flux as in the solar neighborhood. Kulsrud (1978) observes that, when $(\delta \mathrm{B} / \mathrm{B})$ becomes large enough, the damping mechanism saturates, because thermal particles tend to get trapped by the beat wave, instead of continously exchanging energy with it. It turns out that the wave energy densities built up by the cosmic rays in the coronal medium are always high enough to ensure that this saturation effect sets in ; in that case, we obtain : $\quad \Delta \mathrm{v} \simeq 2.5 \varepsilon^{0.8} \mathrm{~T}_{6}^{0.66}\left(\mathrm{~B}_{\mu} \mathrm{n}_{-3}\right)^{0.33} \mathrm{~L}_{1}^{-0.33} \mathrm{Km} \mathrm{s}^{-1}$

We expect the mean age $\mathrm{T}$ to be proportional to $\left(\mathrm{v}_{\mathrm{A}}+\Delta_{\mathrm{V}}\right)$. Thus, formulae (2) or (3) give too steep a rigidity dependence of $\Delta v$ to reconciled with the observations; also, even in the saturated case (formula 3 ), the streaming speed attains a value as high as $(c / 3)$ at a rigidity $\varepsilon \simeq 5 \times 10^{5} \mathrm{GV}$, while the observed anisotropy of cosmic rays in this range of $\varepsilon$ does not exceed $0.1 \%$. We conclude that, if cosmic ray propagation in the interstellar medium is governed by resonant wave-particle interactions in the coronal phase of the interstellar medium, the observations are easier to understand if the waves are due to an underlying interstellar spectrum rather than generated by the cosmic rays themselves (Cesarsky 1975, 1980).

\section{REFERENCES}

Cesarsky, C.J. 1975, Int. Cosmic Ray Conf., 14th, Munich, 12, 4166

Cesarsky,C.J. 1980, to appear in Vol. 18 of Ann.Rev.Astron.Astrophys.

Garcia-Muñoz, M., Mason, G.M., Simpson, J.A. 1977, Ap.J., 217, 859

Kulsrud, R.M. 1978, Astron. p. ded. to B. Stromgren, Copenhagen Univ.Obs.p. 317.

Kulsrud, R.M., Cesarsky, C.J. 1971, Ap.J.Lett., 8, 189

Lee, M.A., Völk, H.J. 1973, Ap. Space Sc., 24, 3I

Mc Cray, R., Snow, T.P., Ann.Rev.Astron.Astrophys., 17, 213

Skilling, J. 1975, M.N.R.A.S., 173, 255

Wentzel, D.G. 1974, Ann.Rev. Astron.Astrophys., 12, 71 